\title{
Epidemiological profile of patients utilizing dental public health services in the eThekwini and uMgungundlovu districts of KwaZulu-Natal province, South Africa
}

SADJ November 2020, Vol. 75 No. 10 p541 - p547

J Mthethwa' ${ }^{1}$, O Mahomed $^{2}$, V Yengopal $^{3}$

\section{ABSTRACT}

\section{Introduction}

Oral conditions such as dental caries, periodontal diseases, tooth loss and trauma affect millions of people globally. These conditions can be prevented when detected early, thereby avoiding adverse outcomes.

The prevalence of oral conditions has been reported in most provinces. However, there is a paucity of data in KwaZulu-Natal.

\section{Aim and objective}

This study aimed to determine the prevalence of oral conditions at public health facilities in two health districts in KwaZulu-Natal, South Africa.

\section{Materials and methods}

A cross-sectional analytical study design was used to determine the nature of oral conditions treated in dental facilities in the eThekwini and uMgungundlovu districts, in KwaZulu-Natal. The study was conducted over a 5-month period (November 2018 - April 2019).

Author affiliations:

1. Jimmy M Mthethwa: BDS, $M P H, M B A$, Department of Public Health Medicine, School of Nursing and Public Health Medicine, UKZN, Durban, South Africa.

ORCID Number: 0000-0002-3586-1930

2. Ozayr H Mahomed: $M B C H B, M M e d, P h D$, Public Health Medicine Specialist, Department of Public Health Medicine, School of Nursing and Public Health Medicine, UKZN, Durban, South Africa. ORCID Number: 0000-0001-8076-0453

3. Veerasamy Yengopal: $B D S, M D e n t, P h D$, Community Dentistry Specialist and Head of Department, Department of Community Dentistry, School of Oral Health Sciences, Wits University, Johannesburg, South Africa.

ORCID Number: 0000-0003-4284-3367

\section{Corresponding author: Jimmy M Mthethwa}

Department of Public Health Medicine, School of Nursing and Public

Health Medicine, UKZN, Congella Rd, Durban, South Africa.

Email: jmmmthethwa@gmail.com

Author contributions:

1. Jimmy M Mthethwa: Study design, data collection, data analysis and write-up of manuscript - $60 \%$

2. Ozayr H Mahomed: Study design, data analysis and write-up of manuscript - 30\%

3. Veerasamy Yengopal: Write-up of manuscript, data analysis - 10\%

\author{
ACRONYM \\ ANUG: Acute Necrotizing Ulcerative Gingivitis \\ BREC: Biomedical Research and Ethics Committee of the \\ University of KwaZulu-Natal \\ DoH: Department of Health \\ DALYs: Daily Adjusted Life Years \\ HIV: $\quad H u m a n$ Immunodeficiency Virus \\ KZN: KwaZulu-Natal \\ SPSS: Statistical Package for the Social Sciences \\ TDI: $\quad$ Traumatic Dental Injuries \\ YLDs: $\quad$ Years Lived with Disability
}

After clinicians examined the patients they entered the data in the patients clinical records and selected information (routine medical history questions as well as 16 possible diagnosis codes) were entered into a data extraction template. The data were analyzed using statistical software SPSS.

\section{Results}

Dental caries was the most prevalent oral condition at $66.4 \%$ followed by periodontal disease at $11.7 \%$, trauma at $7.3 \%$ and lastly tooth loss at $5.9 \%$.

\section{Conclusion}

These findings contribute to knowledge on the prevalence of these oral conditions found in the province.

\section{Keywords}

Dental caries, periodontal diseases, trauma, tooth loss, prevalence.

\section{INTRODUCTION}

According to estimates from the 2013 global burden of disease study, oral diseases affected 3.9 billion people between 1990 and 2010. ${ }^{1}$ In terms of disease burden untreated dental caries in permanent teeth ranked $10^{\text {th }}$ with a combined global prevalence of $35 \%$, whilst periodontal disease ranked $6^{\text {th }}$ with a prevalence of $11 \%$, whereas tooth loss ranked 36th with a global prevalence of $2 \% .^{1}$ 
Oral conditions combined accounted for 15 million daily adjusted life years (DALYs) globally, 1.9\% of all years lived with disability (YLDs), $0.6 \%$ of all DALYs), implying an average health loss of 224 years per 100,000 population. DALYs due to oral conditions increased $20.8 \%$ between 1990 and 2010, mainly due to population growth and ageing. ${ }^{1}$

Dental caries is the most common oral condition and occurs mostly in the occlusal and buccal surfaces of teeth. More than $90 \%$ of dental caries remains untreated in third world countries. ${ }^{2}$ In South Africa, various studies have reported on the prevalence of dental caries over the years. Cleaton-Jones and Fatti reported a predominantly downward trend in the prevalence of caries in Africa particularly in the 5-6 year and 35-44 age groups despite evidence of increased sugar consumption. ${ }^{3}$

Findings from the 2001 South African Oral Health Survey found that almost $60 \%$ of 6 -year-old children had dental caries which was above the $50 \%$ target set by the Department of Health (DoH).4 Furthermore, $80 \%$ of all dental caries in children in South Africa went untreated. $^{4}$

Periodontal diseases include gingivitis, periodontitis, acute necrotizing ulcerative gingivitis (ANUG), HIV-related periodontitis and many other forms. Less than $15 \%$ of children aged 6-12 years old in South Africa presented with healthy periodontal status. ${ }^{4}$ It is well known that oral health affects general health by causing pain and suffering from various oral diseases.

Oral health affects a person's physical and psychological wellbeing. For an example, severe dental caries in children affects their quality of life, as they experience pain, discomfort, disfigurement, eating and sleep disruptions, loss of school days and barriers to learning. Dental caries affects nutrition, growth and weight gain. ${ }^{5}$

The South African draft National oral health strategy document makes the point that the high cost of dental care, the impact it has on oral and general health and its subsequent effect on quality of life warrants that a paradigm shift be adopted in terms of policies and strategies to reduce the burden of oral disease present in our communities.

In KwaZulu-Natal (KZN), oral health services constantly experience challenges of poor prioritization, under-funding, and limited resource allocation when compared to general health services. This has caused some health facilities to report experiencing shortages of consumables, equipment and supplies, inadequate diagnostics and treatment options, long waiting lists and ultimately poor quality of health services.

In addition, there is a paucity of data with respect to the extent of the burden of oral diseases within KwaZuluNatal. The last oral health survey in South Africa was conducted in 2001. It is therefore imperative to identify the epidemiology profile and prevalence of oral diseases in order to inform planning and evidence-based budgeting for oral health services in KwaZulu-Natal and ultimately South Africa.
This study sought to provide information on the epidemiology of some of the common oral conditions at public health facilities in two health districts in KwaZulu-Natal province, South Africa. The objectives of this study were to determine the prevalence of common oral diseases (dental caries, tooth loss, periodontal disease and trauma) among all patients attending dental clinics at public health facilities in the eThekwini and uMgungundlovu districts in KwaZulu- Natal province over a five-month period.

\section{MATERIALS AND METHODS}

This cross-sectional analytical study was designed as an audit of patient attendance and an oral disease profile over a 5 month period (November 2018 to end of March 2019) at public health facilities in the eThekwini and uMgungundlovu districts in KwaZulu-Natal province.

These two districts were selected, as when combined, they served more than $40 \%$ of the entire population of the province. These two districts also represented both urban and semi urban parts of the province and the dental clinics themselves were a combination of five primary health care facilities, one district hospital, three regional hospitals, two tertiary hospitals and one central hospital.

\section{Sampling}

The estimated combined population size for the two districts was approximately 4 million. ${ }^{6}$ The aim was to recruit a at least $10 \%$ of the population for this study noting that almost $90 \%$ of the population use public dental facilities for their oral health needs. With this in mind, the following plan was implemented:

- The oral health team (dentists \& dental therapists) at the facilities in the targeted districts were invited to a morning workshop where the aims and objectives of the study were discussed.

- The data capture form was reviewed with the clinicians who would be responsible for examining the patients and recording the data in the forms.

- Essentially, the clinicians were provided with training on the use of the ICD codes for the 16 conditions that were recorded on the data capture form. The diagnostic data collection tool also allowed clinicians space to describe any other condition that may not have been covered in the 16 conditions.

- The standard demographic information was also captured for each patient.

- All patients of irrespective of age who attended the out-patient dental and maxillofacial department (between $7 \mathrm{am}-4 \mathrm{pm}$ ) in the two districts as well as after hours in the facilities were included in the study.

- All patients or their designated guardian was required to sign informed consent in order to participate in the study.

- Patients who did not provide informed consent and those attending outside the study period were excluded from the study.

Over the 5-month period, 5600 completed records were available for analysis. 


\section{Data collection}

Every patient who attended the facilities had a routine patient record file, which was opened and completed at the reception area of the clinic. The information collected included demographic variables such as age, gender, facility name, and employment status. The clinician (a qualified dentist and/or dental therapist) consulting the patients completed a standardized data extraction sheet following a routine oral health assessment.

The patient clinical information that was usually recorded for every patient was also transcribed into the data capture form by the clinician. This included information such as the diagnosis of the oral condition/s present in the patient at the time of the visit. These condition/s were recorded using the ICD-10 codes and were merely ticked off on the data capture form. An individual patient could have more than one presenting condition, e.g. caries and periodontal disease and these were ticked off on the data capture form.

No calibration was attempted in terms of the clinicians' diagnoses of the oral conditions present in the individual patients. The intention was to record their diagnoses of oral conditions during their daily routine work.

The collected data were captured and analyzed with the assistance of a biostatistician, using the SPSS statistical program. Descriptive statistics are presented as mean, range, and proportions in graphs and tables. Data reliability was ensured by clinicians going back to clinical records for any corrections. In addition patients extracted records which contained missing diagnostic data was excluded in the data analysis.

The Chi-Square Test was used to determine statistically significant associations between age, type of facility, district and most prevalent diagnosis. A 95\% confidence level was adhered to for all statistical tests. A $p$-value of less than 0.05 was considered statistically significant.

Ethical approval was obtained from the Biomedical Research and Ethics Committee of the University of KwaZulu-Natal, reference number (BREC 386/18). Permission, consent and access to the health facilities to conduct the study were obtained from the Provincial Department of Health.

\section{RESULTS}

At the end of the 5-month study period, 5600 patients who had provided consent and received care in the facilities in both districts were included in the study and their clinical data record was included in the data analysis. Most of the patients presenting for oral health care at the identified health facilities in both districts were within the age group of $18-59$ years $(n=4352$, $77.7 \%$ ) (Table 1). The mean age of the patients was 33 years with an age range between 1 and 98 years. The majority $(77.7 \%)$ of patients were between 18 and 59 years of age. Most of the patients were females $(55 \%)$ and $45 \%$ were males. Most of the study patients were from eThekwini district (77\%).
All the facilities in both districts were in urban and semiurban areas. More than three quarters (76\%) of the patients had achieved a greater than secondary level of education and 95\% of them reported having access to clean water at home (Table 1).

\begin{tabular}{|c|c|c|c|}
\hline \multicolumn{2}{|l|}{ Variable } & Number & $(\%)$ \\
\hline \multicolumn{2}{|c|}{ Sample size $\mathbf{N}$} & 5600 & 100 \\
\hline \multirow[t]{3}{*}{ Age (years) } & $0-17$ & 764 & 13.6 \\
\hline & $18-59$ & 4352 & 77.7 \\
\hline & $\geq 60$ & 484 & 8.6 \\
\hline \multicolumn{2}{|l|}{ Sex (F, n (\%) } & 2996 & 54 \\
\hline \multirow{2}{*}{$\begin{array}{l}\text { Name of } \\
\text { District }\end{array}$} & eThekwini & 4329 & 77 \\
\hline & uMgungundlovu & 1271 & 23 \\
\hline \multicolumn{2}{|c|}{ Urban/Semi urban } & 5600 & 100 \\
\hline \multicolumn{2}{|c|}{ >Secondary school education } & 4267 & 76 \\
\hline \multicolumn{2}{|c|}{ Unemployed } & 3681 & 66 \\
\hline \multicolumn{2}{|c|}{ Access to water } & 5316 & 95 \\
\hline
\end{tabular}

\section{Overall prevalence}

The results from this study showed that of the total sample of 5600 patients, dental caries was the most prevalent oral condition at $66.4 \%$ (3719) (Figure 1), followed by periodontal disease at $11.7 \%$ (653), trau$\mathrm{ma}$ at $7.3 \%(411)$ and lastly tooth loss at $5.9 \%$ (331).

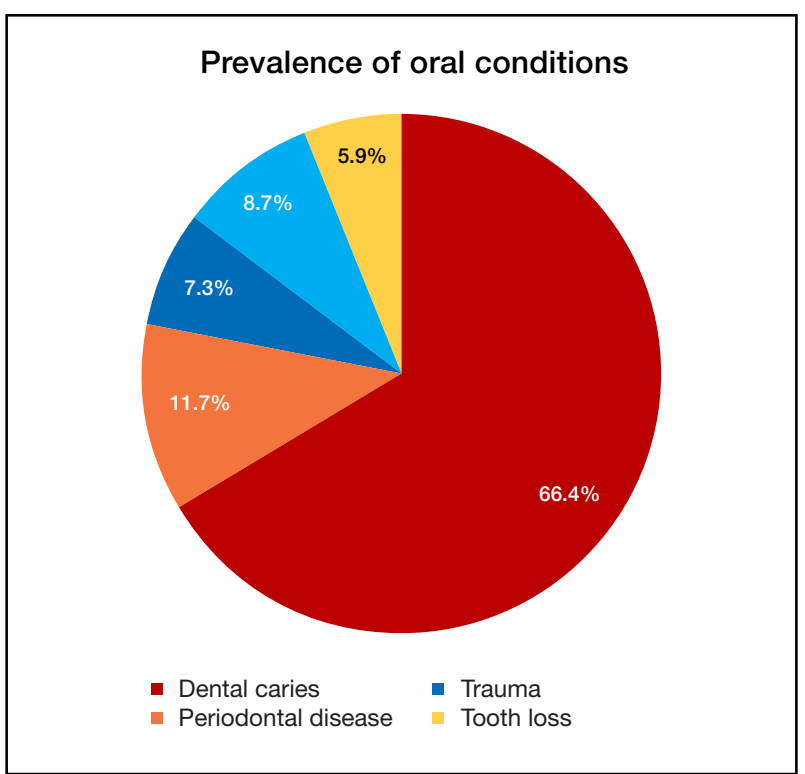

Figure 1. Combined prevalence of oral conditions in the eThekwini and uMgungundlovu districts of KwaZulu-Natal, South Africa.

\section{Dental caries}

Three thousand, seven hundred and nineteen patients (66.4\%) presented with dental caries (Figure 1). Dental caries was significantly higher among the adult population of $18-59$ age group at $85 \%$ (3163) $p<0.001$ compared to the rest of the age categories (Table 2).

Females had significantly higher dental caries rates of $56 \%$ (2092) $p<0.001$ compared to males, whilst eThekwini district had significantly greater proportion of dental caries at $71 \%$ (2639) $p<0.001$ compared to uMgungundlovu district. 


\section{Periodontal disease}

Six hundred and fifty-three patients (11.7\%) presented with periodontal disease (Figure 1). Periodontal disease was significantly higher among the adult population of $18-59$ age group at almost $78.6 \%$ (600) $p<0.001$ compared to the other age categories (Table 2).

Females had higher periodontal diseases rates of (373) $57 \% \mathrm{p}<0.001$ when compared to males, whilst eThekwini district had significantly greater proportion of patients with periodontal disease (653) 96.6\% $\mathrm{p}<0.001$ compared to uMgungundlovu district.

\section{Trauma}

Four hundred and eleven patients presented with trauma (411) $7.3 \%$ (Figure 1). Trauma was significantly higher $62.7 \%(282) \mathrm{p}<0.001$ in the younger age group of $0-17$ compared to the other age groups (Table 2). Trauma was found to be significantly higher levels among males (268) $65.2 \% p<0.001$ compared to females. There were significantly higher levels of trauma $85.1 \%$ (411) $p<0.001$ in eThekwini compared to uMgungundlovu.

\section{Tooth loss}

Three hundred and thirty one (331/5.9\%) patients presented with tooth loss. Figure 1. There was a significant high levels of tooth loss in the 18-59 age group at $48.6 \%$ (161) $p<0.001$ compared to the other age group (Table 2). Males were found to have significantly higher levels of tooth loss $56.5 \%$ (187) $\mathrm{p}<0.001$ compared to females. Significantly higher levels of tooth loss were found in eThekwini 59.8\% (198) $p<0.001$ compared to uMgungundlovu district.

\section{DISCUSSION}

This study provided an epidemiological profile of oral diseases among patients who attended dental facilities in the eThekwini and uMgungundlovu districts of KwaZulu-Natal over a 5-month period. It is worth noting that there was enormous co-operation from both staff and patients involved in this study.

Although we did not track non-respondents, meetings with the clinicians involved in the data collections indicated that very few (less than 5\%) of patients who attended for treatment at the study facilities did not give consent for their information/records to be used in this study.
The KwaZulu-Natal Department of Health annual report described the headcount for clinic attendance in previous years in these districts to have been approximately 2000 per month. ${ }^{7}$ This study managed to capture 5600 patient's records who visited the facilities in 5 months.

The results of this study provide an interesting picture of the prevalence of oral diseases in the eThekwini and uMgungundlovu districts of the KwaZulu-Natal province of South Africa, its distribution across the 3 age ranges and the profile of patients seen in these public oral health facilities.

Dental caries remains the most common oral disease in KwaZulu-Natal province which is consistent with reports on the occurrence of oral disease in other provinces in South Africa. ${ }^{8}$ This is also supported by a myriad of stu-dies undertaken in both developed and developing countries which showed a mixture of high prevalence on dental caries as well as a constant presence of this chronic condition. ${ }^{9-11}$

The results indicate that patients sought dental treatment primarily for the relief of pain associated with dental caries. This was based on their main complaint of dental caries being the highest diagnosis recorded. This finding is consistent with other studies. ${ }^{4,12}$

Furthermore, it also provides evidence that although dental treatment at most of these facilities was free or heavily subsidized, patients continued to access treatment only in the symptomatic phases of the disease when pain becomes the driving force that they seek relief.

Dental caries is a slow progressive disease that can easily be managed in the early stages which can result in the restoration that will save the tooth. There appears to be either an attitude or knowledge (poor attitude and knowledge) problem from the patient's perspective or an access and type of service problem from the facility perspective.

The implication of these findings is that the prevalence of dental caries might actually be increasing in the 18-59 and $>60$ age group and is contrary to the results obtained in the 2013 global prevalence of dental caries study. ${ }^{1}$

The prevalence of dental caries in this study also differs from the global statistics as estimated during the global review which found the prevalence of dental caries among the adult population to be $35 \% .{ }^{13}$

\begin{tabular}{|c|c|c|c|c|c|c|c|c|c|}
\hline \multicolumn{2}{|c|}{ Variables } & \multicolumn{2}{|c|}{ Dental caries } & \multicolumn{2}{|c|}{ Periodontal disease } & \multicolumn{2}{|c|}{ Trauma } & \multicolumn{2}{|c|}{ Tooth loss } \\
\hline \multicolumn{2}{|c|}{ P values } & \multicolumn{2}{|c|}{$<0.001$} & \multicolumn{2}{|c|}{$<0.001$} & \multicolumn{2}{|c|}{$<0.001$} & \multicolumn{2}{|c|}{$<0.001$} \\
\hline \multicolumn{2}{|c|}{ N/\% scores } & Number & Percent & Number & Percent & Number & Percent & Number & Percent \\
\hline \multirow[t]{3}{*}{ Age } & $0-17$ & 233 & 6.3 & 53 & 8.1 & 282 & 62.7 & 37 & 11.2 \\
\hline & $18-59$ & 3163 & 85.0 & 600 & 78.6 & 129 & 31.4 & 161 & 48.6 \\
\hline & $>60$ & 323 & 8.7 & 153 & 13.2 & 68 & 5.8 & 133 & 40.2 \\
\hline \multirow[t]{2}{*}{ Sex } & Male & 1627 & 43.7 & 280 & 42.9 & 268 & 65.2 & 187 & 56.5 \\
\hline & Female & 2092 & 56.3 & 373 & 57.1 & 143 & 34.8 & 144 & 43.5 \\
\hline \multirow[t]{2}{*}{ District } & eThekwini & 2639 & 71.0 & 653 & 96.6 & 411 & 85.1 & 198 & 59.8 \\
\hline & uMgungundlovu & 1080 & 29.0 & 43 & 3.4 & 189 & 14.9 & 133 & 40.2 \\
\hline
\end{tabular}


The combined prevalence of dental caries in this study was $66.4 \%$ which was higher than that found in a study done in 2017 in Limpopo province, which assessed the epidemiological profile of patients utilizing public oral health services, and found a $60 \%$ prevalence. $^{8}$ The findings in our study are similar to another recent cross sectional study in 2020 which looked at prevalence of dental caries among prisoners in the 18-75 age group in all $11 \mathrm{KZN}$ districts. This study found a dental caries prevalence rate of $64.34 \% .^{18}$

It also appears from this study that carious lesions increase with age and that they remain problematic in adults as demonstrated here by an increase from around $30.5 \%$ in the younger age group of $0-17$ years to $72.7 \%$ for the $18-59$ age group. One of the specific observations from this study is that all the patients were from urban and semi-urban areas with no rural patients, this is different from other studies which had varying distribution of patients between urban and rural areas.

The number of individuals younger than 18 years in both districts according to the latest population census data is approximately $1000000,26 \% .{ }^{6}$ However, when one examines the patient profile of clinic attendees, it was concerning that children made up only $15 \%$ (856 out of the 5600) of the total number that visited dental health facilities. When one compares the prevalence rates of caries among children as reported in the children's national oral health survey, there is evidence, for example, that more than $50 \%$ of children under the age of 15 years have tooth decay. ${ }^{4}$

This implies that children should form a much larger percentage of the population that seek care for treatment of dental caries. However, out clinic attendance rate of $15 \%$ shows that children are either not attending or access is a problem especially when one considers that free care is available for this group at most dental clinics in the public sector in KZN. The overall findings from this study has important implications for oral health planning in the province.

\section{Periodontal disease}

Periodontal disease in this study was found to have a prevalence rate of $11.7 \%$ which was similar to the study done in 2017 in Limpopo which also found a 11.0\% prevalence of periodontal disease. ${ }^{8}$ In a systematic review of the global epidemiology of dental caries and severe periodontitis conducted in 2017, periodontitis was the sixth most prevalent condition and affected $10.8 \%$ or 743 million people aged $15-99$ years worldwide.

This study reported that severe periodontitis in the global population had remained static over the previous two decades at $11.2 \%$ in 1990 and $10.5 \%$ to $12.0 \%$ in 2010.13 The results of this systematic review used a more robust methodology of age-standardized methods and adds support to our findings.

In contrast the national oral health survey found a lower prevalence of $8.3 \%$ for periodontal disease. The differences in these findings could be partly explained by the fact that the present study allowed clinicians' discretion and clinical judgment to define periodontal diseases as the presence of both or either gingival and periodontal diseases whereas the national survey used the presence of deep pockets as a proxy for periodontal disease. It is worth noting that there are other international surveys which demonstrated that the prevalence of pocketing of $6 \mathrm{~mm}$ or more is between $5 \%$ and $20 \%$ for much of the world's population. ${ }^{14}$

A recent study conducted in 2019 in KZN exploring oral health status of pregnant woman found evidence of periodontal disease related conditions such as gingival inflammation, pregnancy epulis 8,5\%, oral lesions $14.7 \%$, and tooth mobility $5.9 \% .{ }^{19}$ Early oral health screening during pregnancy can ensure the overall well-being of both the mother and the foetus.

Another national survey of employed adults found the prevalence of gingival bleeding was $44 \%$, the prevalence of pocketing of $4 \mathrm{~mm}$ or more was $14 \%$, and the prevalence of attachment loss of $3 \mathrm{~mm}$ or more was $44 \%$. This study also found that there are other risk indicators for a higher prevalence of periodontal disease which include increasing age, poor education, lack of professional dental care, previous periodontal destruction, tobacco use, and diabetes. ${ }^{14}$ In support of this study, our study also found a significant association between age and periodontal diseases. This study found a $78.6 \%$ prevalence of periodontal diseases among the 18-59 years adult population $p<0.001$ compared to the other age groups.

\section{Trauma}

This study found a prevalence of $7.3 \%$ for dental and maxillofacial trauma, which is similar to an average of $8 \%$, reported in a study conducted in Johannesburg in the year 2000, in patients treated for maxillofacial injuries. ${ }^{4}$ This study further found that of the $8 \%$ injuries, $48 \%$ were as a result of fights, assaults and gunshots. A limitation of our study is that trauma cases were pooled together and the reasons for the trauma were not listed. A study which was done in 2017 looked at the profile of 5 district hospitals providing oral health in Limpopo and found a $7 \%$ prevalence of patients with dental trauma in 500 patients clinical charts.

It further found a $4 \%$ prevalence of dental trauma in the younger group under the age of 19 years. ${ }^{8}$

One of the possible explanations for our study finding a slightly higher prevalence of trauma could be partly explained by the inclusion of both dental and maxillofacial trauma, as opposed to the Limpopo study which seem to have looked at only dental trauma. Another possible explanation is that the two biggest referral maxillofacial centers in the KwaZulu-Natal province are located within the two districts of our study focus.

Our study also included the three largest tertiary referral health facilities in KwaZulu-Natal, namely, Greys Hospital, King Edward and Inkosi Albert Luthuli Central Hospital in the sample as opposed to the Limpopo study which only looked at district hospitals. 
Our study also found a significant difference in trauma among the males and females as well as the two districts. eThekwini had the higher prevalence rate $85,1 \%, p<0.001$. Both districts are urban and semi urban populations, a possible explanation for a higher prevalence in eThekwini could be purely as a result of a higher population in eThekwini almost 4000000 compared to uMgungundlovu almost 1000000 population, which means that eThekwini had almost 3 times more people than uMgungundlovu. ${ }^{6}$

There are many studies that support the notion that males are more at risk of trauma compared to females, possible explanation of the gender differences is the risky behavior that males often engage in, however it is difficult to explain the reason for this study finding higher rates of trauma in the younger age group. One possible explanation could be that the definition of trauma in this study allowed for both dental and maxillofacial trauma. It is possible that part of the trauma related to falls, tooth trauma, assaults and injuries.

A study of traumatic dental injuries (TDI) among 4-15 years old children in India found a prevalence of $4.15 \%$. The maxillary anterior teeth accounted for $95.45 \%$ of the TDI injuries. Maxillary central incisor was the most common tooth to be affected due to trauma (54.5\%). Enamel with dentin fracture with pulp exposure was the main type of TDI (43.1\%). ${ }^{15}$ Although our study did not differentiate the type of injuries like the India study, our findings are similar to this study if you combined their findings according to the various types of injures.

Literature suggest that TDI are common in children. ${ }^{16}$ There are few data on prevalence of TDI in South African populations. In a south African study that assessed TDI of permanent incisors in 11 to 13-year-old South African schoolchildren the prevalence of TDI was $6.4 \%$. Falls were the main cause of TDls, playing sports was the second most common cause and the third most common cause was collision with objects. ${ }^{16}$ Overall the findings of our study are similar to other studies. Our study did not differentiate the type of injuries and grouped together both dental and maxillofacial injures. We would suggest that future studies could explore this further in this age group of $0-17$ years as to the reasons for the injuries.

\section{Tooth loss}

This study collected data from the various age groups in order to explore tooth loss among the different age groups. This study found an overall prevalence of tooth loss at $5.9 \%$. Tooth loss is often associated with advancing age as such our study explored this phenomenon and found that the 18-59 years group had a prevalence of $48.6 \%$ and the 60 years and above age group had a prevalence of $40.2 \%$.

The Van Wyk study found an overall prevalence of tooth loss to be $12.6 \% .^{4}$ Van Wyk study also found that there were other regions within provinces such as the Western Cape, which had a prevalence, as high as $51.6 \%{ }^{4}$ The high number of tooth loss was also found in a recent cross sectional study in 2020 which looked at prevalence of missing teeth among prisoners in the 18-75 age group in all $11 \mathrm{KZN}$ districts. This study found a tooth loss prevalence rate of $71,85 \% .^{18}$ This supports the notion that some categories of the population can have very high tooth loss rates.

It would seem from these findings that KwaZulu-Natal had the lower prevalence of $5.9 \%$ compared to the national average of $12.6 \%$. This is an important finding in this study that needs to be explored further as to the reasons for the lower prevalence, what are some of the protective factors that may be contributing to this fact.

There seem to be global differences in tooth loss. The findings in this study as well as the Van Wyk study showed a higher prevalence of between 5.9-12.6\%. In contrast to this, there are other global studies done in a 2014 systematic review of global burden of severe tooth loss, which reported a general global prevalence of $2.5 \%$ tooth loss. ${ }^{17}$

This global prevalence represented both developed and developing countries. We would like to explore possible factors such as diet, oral hygiene status, health system, socioeconomic status that may be possible factors to these differences in tooth loss.

Another factor worth noting in our study is that the data collected were not disaggregated into the various racial groups in south Africa, whereas the last oral health survey had explored tooth loss in the different racial groups in south Africa. In doing so it found that the colored community had as high as $51 \%$ prevalence. There is anecdotal knowledge of the specific cultural behavioral phenomenon reported in some parts of the colored community in the western cape such as deliberately removing the front teeth, thus contributing to high levels of tooth loss, it would be of interest to explore tooth loss and this racial behavioral aspect in future studies and compare to the van Wyk study.

The overall contribution of this study in the tooth loss debate suggests that although $\mathrm{KZN}$ had a lower rate than the National rate, tooth loss remains a health challenge among the adult population in both the 18-59 and above 60 years age groups. This challenge needs to be addressed, one possible explanation is that there may be a relationship between the high prevalence of dental caries as demonstrated in this study, patients maybe seeking treatment late and resorting to clinicians performing dental extractions treatment which in turn also contributes to the tooth loss phenomenon in the adult population.

\section{Limitations}

The main limitation of this study is that oral conditions were diagnosed according to the clinicians' perspective, i.e., there was no attempt to standardize diagnostic criteria and no calibration of clinicians' were undertaken. This could have led to an over or under-estimation of the disease profiles reported. It may be interesting to compare these oral disease profiles against one in which clinicians were calibrated and standardized for diagnos- 
ing common oral conditions. It would provide evidence of an over/under estimation of oral diagnoses in this setting.

In terms of trauma data collected in this study, selection bias could have been possible as the three largest dental and maxillofacial referral facilities in the entire province reside within the study population, this may have affected the trauma prevalence indicator. This study also attempted to examine the data based on the type of facility, it was noticed that all the facilities which responded were based in urban and semi-urban areas.

As such it was not possible to include the rural vs. urban prevalence data in this study. It may be useful in future studies to compare rural and urban settings against these 4 most prevalent conditions found in this study. Another limitation of our study is that trauma cases were pooled together irrespective of the type of trauma. This may affect the comparison of trauma prevalence rates in literature, it may be useful in future studies to explore the trauma indicator in more detail.

\section{CONCLUSION}

The findings of the current study contributed to knowledge in the province in determining the four most prevalent conditions as serious dental public health challenges facing the major urban and semi-urban population in KwaZulu-Natal.

The findings have also pointed out the disease profiles affecting both males and females as well as eThekwini and uMgungundlovu districts. It is hoped that these findings will therefore be useful to policy makers to develop more responsive preventive and dental treatment services plan for the adult population of KwaZulu-Natal.

\section{Acknowledgements}

This was a self-funded study. The authors wish to acknowledge the KwaZulu-Natal Department of health for giving permission to use its platform to conduct this study as well as the $\mathrm{KZN}$ DoH staff at all participating facilities.

We wish to also acknowledge the KZN DoH Head office staff for active participation in the data collection. We are grateful for the scholarly inputs provided by colleagues at the University of Witwatersrand and School of Oral Health Sciences.

\section{References}

1. Marcenes.W, Kassebaum.JN, Bernabé. E et al. Global burden of oral conditions in 1990-2010: a systematic analysis. Journal of Dental Research. 2013; 92(7): 592-7

2. Kathmandu RY. The burden of restorative dental treatment for children in Third World countries. International Dental Journal. 2002; 52(1): 1-9.

3. Cleaton-Jones P, Richardson BD, Granath L et al. Nutritional status and dental caries in a large sample of 4-and 5-year-old South African children. SAMJ. 2000; 90(6): 631-5.

4. Van Wyk PJ, Van Wyk C. Oral health in South Africa. International Dental Journal. 2004; 54(6): 373-7.

5. Sheiham A. Oral health, general health and quality of life. Scielo Public Health, Bulletin of the World Health Organisation. 2005; 83(9): 644-5

6. Statistics South Africa. Statistics by place/Metropolitan Municipality 2017, July [Available from: http://www.statssa.gov. za/?page_id=1021\&id=ethekwini-municipality].

7. KwaZulu-Natal Department of Health. Annual report 2018 [Available from: http://www.kznhealth.gov.za/2017-2018-Annual-Report.pdf].

8. Thema LK, Singh S. Epidemiological profile of patients utilising public oral health services in Limpopo province, South Africa. African journal of primary health care \& family medicine. 2017; 9(1): 1-5

9. Tobin AO, Ajayi O. Common oral conditions and correlates: an oral health survey in Kwara State Nigeria. BMC. 2017; 10(1): 1-9

10. Petersen P. The World Oral Health Report 2003: Continuous improvement of oral health in the $21^{\text {st }}$ century-the approach of the WHO Global Oral Health Programme.Community and Oral Epidemiology Journal. 2003; 31: 3-24.

11. Petersson GH, Bratthall D. The caries decline: a review of reviews. European Journal of Oral Sciences.1996; 104(4): 436-43

12. Muwazi LM, Rwenyonyi CM, Tirwomwe FJ et al. Prevalence of oral diseases/conditions in Uganda. African journals online. 2005; 5(3): 227-33.

13. Frencken JE, Sharma P, Stenhouse $L$ et al. Global epidemiology of dental caries and severe periodontitis - a comprehensive review. Journal of Clinical Periodontology. 2017; 44(S18): 94-105.

14. Fox $\mathrm{CH}$. New considerations in the prevalence of periodontal disease. Europe PMC.1992; 2: 5-11.

15. Gupta S, Kumar-Jindal S, Bansal M, Singla A. Prevalence of traumatic dental injuries and role of incisal overjet and inadequate lip coverage as risk factors among 4-15 years old government school children in Baddi-Barotiwala Area, Himachal Pradesh, India. Med Oral Patol Oral Cir Bucal Journal. 2011; 16(7): 960-5

16. Naidoo S, Sheiham A, Tsakos G. Traumatic dental injuries of permanent incisors in 11- to 13-year-old South African schoolchildren. Dental Traumatology Journal. 2009; 25(2): 224-8.

17. Marcenes W, Flaxman A, Kassesbaum N et al. Global Burden of Oral Conditions in 1990-2010: A Systematic Analysis. Journal of Dental Research. 2013; 92(7): 592-7.

18. Radebe M, Singh S. Investigating dental caries rates amongst sentenced prisoners in KwaZulu-Natal, South Africa. South African Dental Journal. 2020 Apr; 75(3): 137-41.

19. Africa CW, Turton M. Oral health status and treatment needs of pregnant women attending antenatal clinics in KwaZulu-Natal, South Africa. International journal of dentistry. 2019 Mar 4; 2019. 